Monatsschrift f. Geburtshülfe u. Gynäkologie 1917;46:268-271

\title{
Julius Schottländer †
}

Von E. Wertheim

Am 29. Mai d. J. ist Professor Schottländer, der wissenschaftliche Assistent und

Laboratoriumsleiter an der II. Frauenklinik in Wien, infolge eines Herzschlages in Kiel, wo er seit Kriegsbeginn als kgl. Marine-Oberstabsarzt der Reserve tätig war, verschieden.

Die Gynäkologie erleidet damit einen schweren Verlust. Schottländer war in der Histologie und pathologischen Anatomie der Frauen-krankheiten eine allgemein anerkannte Autorität, und manche seiner Arbeiten auf diesem Gebiete haben bleibenclen Wert. In der Erforschung der Genitaltuberkulose war er wegweisend, seine Publikation über die von den Genitalien ausgehenden und in den Genitalien lokalisierten Metastasen ist die erste zusammenfassende Darstellung des Gegen-standes in großem Stile, eine Fundgrube der Belehrung und infolge der eingehenden Berücksichtigung der Literatur von hohem Werte für künftige Bearbeitungen, sein Werk über das Uteruskarzinom (gemeinsam mit Kermauner) eine in ihrer Art einzig dastehendeLeistung, von welcher Robert Meyer sagt, daß es eine ähnlich eingehende und sorgfältige Be-arbeitung nicht nur auf dem Gebiete des Uteruskrebses nicht gibt, sondern überhaupt von keiner Geschwulst. Infolge der klaren Diktion, des Gedankenreichtums, der Übersichtlichkeit und plastischen Darstellung gestaltet sich das Studium der Schottländerschen Schriften äußerst lehrreich. Schottländer war ein glänzendes Beispiel dafür, wie man von einer ganz speziellen Arbeitsrichtung aus neue und frucht-bringende Gesichtspunkte für das ganze Fach zu eröffnen imstande ist. Obwohl er sich fast ausschließlich mit histologischen Forschungen be-faßte, war er doch ein nach alien Richtungen geschulter Kliniker mit sçharfem Blick für alle neu auftauchenden Fragen, zu denen er von Julius Sohottlander $\uparrow$.

269

seinem speziellen Forschungsgebiete aus in aktueller Weise Stellung zu nehmen pflegte.

Die Arbeiten Schottländers sind:

Über Kern- und Zellteilungsvorgänge am Endothel der entzündeten Hornhaut. (In.-Diss.) Arch. f. mikr. Anat. 1888. 31.

Beitrag zur Kenntnis der Follikelatresie nebst Bemerkungen über die unveränderten Follikel in den Eierstöcken der Säugetiere. Arch, f. mikr. Anat. 1891. 37. S. 192.

Über die Entstehung des Graafschen Follikels beim Menschen und seinen Untergang bei Mensch und fe $1 /$ sugetier. Ztschr. f. Geb. u. Gyn. 1892. 24. S. 312.

Dasselbe Thema. Zbl. f. Gyn. 1892. S. 440.

Über die drüsigen Elemente in Fibromyomen des Uterus. Ztschr. f. Geb. u. Gyn. 1893. 27. S. 321.

Über den Graafschen Follikel, seine Entstehung beim Menschen und seine Schicksale bei Mensch und Tier. Arch. f. mikr. Anat. 1893. 41. (Habilitations-Schr.)

Über die Tuberkulose des Eierstockes und der Eierstocksgeschwülste nebst einigen Bemerkungen über die Tuberkulose des Eileiters. Monatsschr. f. Geb. u. Gyn. 1897. V. S. 321 u. 448. 
Über Eierstockstuberkulose. Jena 1897. G. Fischer.

Kasuistischer Beitrag zur Lehre von der Osteomalacie. Ztschr. f. Geb. u. Gyn. 1897. Bd. 37. H. 3.

Über mehreiige Follikel und mehrkernige Eizellen. Monatsschr. f. Geb. u. Gyn. 1904. 20. 1187 u. Monatsschr. f. Geb. u. Gyn. 1905. 21. S. 622.

Zur histologischen Diagnose von Frühstadien der Uterustuberkulose. Monatsschr. f. Geb. u. Gyn. 1905. 21.

Uterus bicornis unicollis mit Vagina subsepta und Cystenbildung, mit Drüsenwucherung im Gebiet des linken cervikalen und vaginalen Gartner-Gang-Abschnittes. Arch. f. Gyn. 1906. 81. Beitrag zur Lehre von den Dermoidcysten des Eierstockes. Arch, f. Gyn. 1906. 78. S. 137. Zur Histogenese der Portio-Erosionen. Monatsschr. f. Geb. 1907. 26

Epikritische Bemerkungen zur Arbeit Gottschalks über die Entstehung der Erosion der Portio vaginalis. Mon. f. Geb. 1909. 30, Heft 2.

Gedenkrede auf weil. Hofrat v. Rosthorn. Wien. klin. Woch. 1909. Xo. 44.

Xachruf für Rosthorn. Dtsch. med. Woch. 1909. No. 37.

A. v. Rosthorn. Arch. f. Gyn. 1909.

A. v. Rosthorn. Wurzbachs biograph. Lexikon.

Kritische Besprechung von Pfannenstiels Erkrankungen der Eier-stöcke in Veits Handbuch.

Monatsschr. f. Geb. 1909. 30. S. 390,

Zur Kenntnis des Uteruskarzinoms. Gemeinsam mit Kermauner* Berlin 1912. S. Karger.

Kurzer Bericht über die während 33/4 Jahren beobachteten malignen blastomatösen

Veränderungen der Uterusmyome. Zbl. f, Gyn. 1912. 8. 656.

270

Julius Schottländer $\uparrow$.

In welcher Weise läßt sich die Frühoperation des Gebärmutter-krebses fördern. Wien. klin.

Woch. 1912. S. 1933.

51/8 Jahre pathologisch-anatomische Tätigkeit im Laboratorium der Universitäts-Frauenklinik

Wien. Naturforscherversammlung 1913.

Über die von den Genitalgeschwülsten des Weibes ausgehenden metastat $1 / 8$ chen Geschwülsten in den übr'gen Körperorganen und die metastatischen Geschülste in den weiblichen Geschlechtsorganen. ÏSiothnagels spez. Path. u. Ther. Suppl. 7. Bd. II. S. 470.

Über histologische Geschwulstdiagnose im Bereich der Gebär-rautter. Arch. f. Gyn. Bd. 100. S. 225.

Über die Bestimmung der Schwangerschaftsdauer auf Grund histo-logischer Placentarbefunde und über etwaige praktische Verwert-barkeit dieser Befunde. Zbl. f. Gyn. 1913. S. 193.

Nochmals: Dasselbe Thema. Zbl. f. Gyn. 1913. S. 1195.

Zur Theorie der Abderhaldenschen Schwangerschaftsreaktion, sowie Anmerkungen über die innere Sekretion des weiblichen Genitales. Zbl. f. Gyn. 1914. S. 425.

•30. Zur histologischen Wertung und Diagnose der Radiumveränderun-gen beim

Uteruskarzinom. Strahlentherapie. 1915. V. S. 604.

Besonders schmerzlich ist sein Tod für die II. Frauenklinik in Wien. Das große Laboratorium derselben hat er in mustergültiger Weise ein-gerichtet und den Betrieb bleibend organisiert.

Seine ganze Arbeits-kraft hat er demselben gewidmet, nicht einmal eine Mittagspause außer-halb desselben gönnte er sich. Die exakte Ordnung und Protokollierung des Materials, die er einführte, war die Yoraussetzung für die von ihm bereits ausgeführten und für die von ihm 
geplanten Arbeiten· Der Urn-fang und die Intensität seiner Laboratoriumstätigkejt läßt sich am besten erkennen aus dem von ihm selbst auf der Naturforscherversamm -lung 1913 erstatteten Berichte über ,,5\% Jahre pathologisch-anatomische Tätigkeit im Laboratorium der II.

Universitäts-Frauenklinik in Wien". Dabei wirkte er äußerst anregend auf seine Schüler, Welche unter seinem Einflusse und unter seiner Leitung eine beträchtliche Anzahl wert-voller Arbeiten ausführten. Einige derselben kommen demnächst zur Veröffentlichung.

Julius Schottländer, geboren am 12. IV. 1860 zu St. Petersburg, studierte in Heidelberg und München und Wurde 1887 in Heidelberg promoviert, sodann War er Assistenzarzt bei F. A. Kehrer in Heidelberg und bei A. Martin in Berlin. 1893 habilitierte er sich in Heidelberg, 1897 Wurde er Extraordinarius und zweiter Examinator. 1903 über-nahm er das Laboratorium der Frauenklinik in Heidelberg, an Welcher damals Rosthorn lehrte, und im Jahre 1908 übersiedelte er mit Rosthorn nach Wien, um die Einrichtung und Leitung des Laboratoriums der II. Frauenklinik zu übernehmen.

In jenen Jahren gemeinsamer Tätigkeit in Heidelberg haben sich innige freundschaftliche Beziehungen zWischen den beiden Männern entwickelt, und Rosthorn war es, der Schottländer zurÜbersiedlung nach Wien beWog. Schottländer hing mit Verehrung und BeWunderung an Rosthorn, an dessen idealem Schwung er sich begeisterte. Schottländer war ja selbst eine Persönlichkeit von rein idealem Streben, der sich mit

Julius Sehottländer $\uparrow .271$

der ganzen Kraft dem frei gewählten Berufe widmete und nicht für Geld und äußere Ehren, sondern für die Wissenschaft und für die Wahr-heit und für die Erfüllung der Forderungen seines eigenen Gewissens arbeitete. Der plötzliche Tod Rosthorns nach nur einjähriger Wirksam-keit in Wien war für Sehottländer der schwerste Schlag. In den Nekro-logen, die er Rosthom widmete, kommt sein Schmerz ergreifend zum Ausdruck. Nur einem günstigen Zusammenwirken verschiedener Um-stände ist es zu danken, daß Sehottländer damals der Klinik erhalten blieb. Hauptsächlich Waren es in Angriff genommene wissenschaftliche Arbeiten und das dazu bereitgestellte Material, was ihn hier fesselte. Die Fertigstellung des großen Werkes über das Uteruskarzinom fällt in diese Zeit.

Als der Krieg ausbrach, litt es Sehottländer nicht länger in Wien; er eilte, seine Dienste dem Vaterlande zur Verfügung zu stellen. Wir rechneten mit Sicherheit auf sein Wiederkommen bei Kriegsende und bedauern tief, daß das Schicksal es anders gefügt hat. Ich habe die feste Überzeugung, daß sich, nachdem ich in den ersten Jahren meiner Klinikführung vollauf damit zu tun gehabt hatte, mich in meinen neuen Wirkungskreis einzuarbeiten, zwischen uns, zum Wohle der Khnik, ein ähnliches Verhältnis entwickelt hätte, wie es zwischen ihm und dem ihm im Tode vorausgegangenen Rosthom bestanden hatte, und ich fühlte es als eine Schuld, die ich Sehottländer gegenüber ab-zutragen hatte, diesem hervorragenden Marine gegenüber alles, was in meiner Macht stand, dazu beizutragen, um ein solches Verhältnis her-zustellen. . Jedenfalls wird sein Wirken an der II. Frauenklinik für uns immer beispielgebend bleiben und wir werden es immer als großes Verdienst Rosthorns betrachten, daß es ihm gelungen ist, diese hervorragende Kraft an die II. Frauenklinik zu ziehen.

Wien, Juni $1917 . \quad$ Professor Dr. E. Wertheim.

Als Sehottländer 1891 sich als mein Mitarbeiter meldete, erlaubte mir die Enge meiner damaligen privaten Anstalt nicht, ihm auf die Dauer einen seinen Fähigkeiten und seinen Wünschen gebührenden Platz einzuräumen. Nach kurzen Monaten kehrte Sehottländer nach Heidelberg zurück. Es war mir eine große Freude, daß Rosthom nach 
seiner Übersiedlung nach Heidelberg mir des öfteren wiederholen konnte, wie zutreffend meine Warme Befürwortung Schottländers seinen vollen Beifall gefunden. In der Tat brachte Sehottländer ein so trefflich solides Wissen für die akademische Arbeit mit, daß man ans eine Forscher- und Lehrleistungen die höchstgespannten Erwartungen anknüpfen konnte. Der so frühe jähe Toci Rosthorns, die Schwierigkeiten des Interregnum, dann der große Existenzkampf der deutschen Kultur und Wissenschaft, sie haben Schottländers Arbeit noch nicht zu der vollen Entwicklung ihrer Früchte gelangen lassen. Da aber, Was er uns schon jetzt hat geboten, läßt erkennen, wie berechtigt die Hoffnungen gewesen sind, die wir daran knüpften. Und nun ist dieser tapfere Streiter uns entrissen! Wir trauern ihm nach, besonders diejenigen, welche unter dem Banne seiner persönüchen Liebenswürdigkeit und seines vornehmen Charakters gestanden haben! A. Martin. 